\title{
Failure Analysis of Gas Turbine Blades
}

\author{
Rybnikov A.I.*, Getsov L.B.*, Leontiev S.A.** \\ * Polzunov Central Boiler and Turbine Institute (NPO CBTI), Saint-Petersburg, Russia \\ ** Turbine-Building Production Association "LMZ", Saint-Petersburg, Russia
}

Different failures of blades made from superalloys may be observed during gas turbine plant testing and operation. The cause of these failures is usually identified both by metallographic methods (microstructural studies, fractography, X-ray crystal analyses), bench and laboratory strength tests, and by strength calculation methods, including non-conventional methods.

Long-term gas turbine operation leads to the structural degradation of superalloy blades - there is a change in a number, shape and size of $\gamma^{\prime}$-phase particles and in carbide amounts, distribution and composition. The formation of close-packed topological phases $(\sigma, \mu, \lambda$-phases) can also be observed. In a number of cases, the structural degradation results in a significant change in the mechanical properties, which can cause blade failures.

The present paper deals with several investigations into the causes of typical blade failures during long-term service.

Static stress failure of gas turbine blades made of superalloys. The industrial production of blades by different manufacturers may involve process violations causing a displacement of the mass center and, as a consequence, the static failure of blades at a fairly high carrying capacity strength margin. Such blade failures were observed in aircraft, marine and stationary gas turbine plants. Thus, 13 cases of rotor blade failures were discovered in a generating gas turbine plant after operation for 1000 to $6000 \mathrm{~h}$. The fractographic study revealed a static cracking mechanism initiated at blade edges. Blade strength calculations were carried out by the finite element method with regard to creep under the conditions of centrifugal forces and mass center displacement. It was determined that, due to the stress increase at edges and the difficulties associated with stress redistribution during creep, the stresses can reach the material long-term strength values corresponding to rupture life.

Another cause of static stress failure of blades is blade overheating related to the departures from normal operating conditions. Such failures are detected by metallographic methods based on metal structural variations throughout the entire blade section or in its separate regions as well as on the formation of thick de-alloyed surface layers. Thus, cracks on ZhS6K-alloy guide blade edges were discovered after GT adjustment testing for $103 \mathrm{~h}$. with 57 start-ups. The metallographic study revealed $\gamma^{\prime}$-phase size growth, which suggests that the operating temperature of metal had risen to about $1050^{\circ} \mathrm{C}$ (instead of the normal $850^{\circ} \mathrm{C}$ operating condition). The analysis showed that this temperature elevation could be caused by the disruption of cooling conditions in the channel (because of the non-removed ceramic rod, casting residues, etc.). The elevated metal temperature resulted in a drastic deterioration of mechanical properties and, above all, in reduced metal fatigue resistance that caused cracking.

Thermal fatigue cracking of rotor and guide blades. Thermal fatigue cracks are the characteristic type of edge failures in gas turbine cooled blades, including those manufactured from single-crystal and directional-solidified alloys. In long-term operation, such cracks also form on blades made from wrought high-temperature alloys. Based on the metallographic studies 
of micro-crack propagation in wrought alloys at elevated temperatures, a diagram has been developed in order to predict a type of alloy rupture depending on the temperature and frequency of cyclic loading. The diagram allows diagnostics to be made of blade damage detected in operation.

Thermal fatigue cracking of rotor and guide blade coatings. Coating cracking is induced by a local corrosion failure of blade base metal under the coating. A method for testing small-size coating specimens has been developed. The method makes it possible to observe strain relief characteristics during testing and to study the mechanisms of crack initiation and propagation in a coating up to specimen failure. A series of thermal fatigue tests was performed using different superalloy specimens with different coatings. The mechanisms of micro-crack initiation and suppression in multi-layer coatings have been determined.

Corrosion and corrosion fatigue cracking of rotor and guide blades. High-temperature sulfideoxide corrosion and high-temperature alloy surface de-alloying processes have been investigated by blade surface metallography (X-ray, EDS spectrum analysis). It has been found that after operation for $100000 \mathrm{~h}$., the uncoated rotor blades made from wrought alloy EI893 experience a decrease in chromium content in a layer up to $100 \mu \mathrm{m}$ thick.

The analysis of surface layer composition in the region of cracks in blades made from hightemperature alloys EI929, ZMI-3 and CNK-7 after operation for 25000 to $35000 \mathrm{~h}$. revealed a local increase in sulfur content related to sulfide-oxide corrosion attack.

The long-term corrosion and de-alloying effects on material and fatigue strength characteristics have been analyzed. Thus, it has been found that corrosion cracking processes develop from blade metal surface exposure to the action of fuel combustion products containing sulfur-rich compounds.

High-temperature alloy and protective coating corrosion life was estimated and the results were compared to the experimental data obtained during blade inspection after long-term operation. The agreement between the results was found to be satisfactory. 